\begin{tabular}{|c|l|}
\hline Title & Correlated-Gaussian approach to linear-chain states: Case of four a particles \\
\hline Author(s) & Suzuki, Y.; Horiuchi, W. \\
\hline Citation & $\begin{array}{l}\text { Physical review C, 95(4), 44320 } \\
\text { https://doi.org/10.1103/PhysRevC.95.044320 }\end{array}$ \\
\hline Issue Date & 2017-0424 \\
\hline Doc URL & http://hdl.handle.net/2115/66294 \\
\hline Rights & ○2017 A merican Physical Society \\
\hline Type & article \\
\hline File Information & PhysRevC95 044320.pdf \\
\hline
\end{tabular}

Instructions for use 


\title{
Correlated-Gaussian approach to linear-chain states: Case of four $\alpha$ particles
}

\author{
Y. Suzuki ${ }^{1,2}$ and W. Horiuchi ${ }^{3}$ \\ ${ }^{1}$ Department of Physics, Niigata University, Niigata 950-2181, Japan \\ ${ }^{2}$ RIKEN Nishina Center, Wako 351-0198, Japan \\ ${ }^{3}$ Department of Physics, Hokkaido University, Sapporo 060-0810, Japan \\ (Received 5 February 2017; revised manuscript received 21 March 2017; published 24 April 2017)
}

\begin{abstract}
We show that correlated Gaussians with good angular momentum and parity provide flexible basis functions for specific elongated shape. As an application we study linear-chain states of four $\alpha$ particles in variation-afterprojection calculations in which all the matrix elements are evaluated analytically. We find possible chain states for $J^{\pi}=0^{+}, 2^{+}, 4^{+}$and perhaps $6^{+}$with the bandhead energy being about $33 \mathrm{MeV}$ from the ground state of ${ }^{16} \mathrm{O}$. No chain states with $J \geqslant 8$ are found. The nature of the rotational sequence of the chain states is clarified in contrast to a rigid-body rotation. The quadrupole deformation parameters estimated from the chain states increase from 0.59 to 1.07 for $2^{+}$to $6^{+}$. This work suggests undeveloped fields for the correlated Gaussians beyond those problems which have hitherto been solved successfully.
\end{abstract}

DOI: 10.1103/PhysRevC.95.044320

\section{INTRODUCTION}

Spatially localized motion of nucleons plays an important role in nuclear structure through excitation mechanism, pairing, $\alpha$ clustering, etc. A spatially localized single-particle (sp) orbit is needed, and is conveniently represented by a Gaussian wave packet (GWP)

$$
\phi_{s}^{v}(\boldsymbol{r})=\left(\frac{v}{\pi}\right)^{\frac{3}{4}} e^{-\frac{v}{2}(\boldsymbol{r}-s)^{2}}
$$

where $s$ denotes the position of the packet and $v^{-1 / 2}$ determines its width or spatial extension. Several acronyms used in this paper are listed in Appendix A. The GWP is widely used in the cluster model and its extended models [1-3], but is not popular in shell-model or configuration interaction calculations, probably because it has no definite orbital angular momentum. As a localized orbit with good angular momentum, we propose a locally peaked Gaussian (LPG) specified by $k$ and $a$,

$$
\varphi_{k l m}^{a}(\boldsymbol{r})=\frac{1}{G_{2 k+l}}\left(\frac{a^{3}}{\pi}\right)^{\frac{1}{4}}(\sqrt{a} r)^{2 k+l} e^{-\frac{a}{2} r^{2}} Y_{l m}(\hat{\boldsymbol{r}}),
$$

where $\hat{\boldsymbol{r}}$ is the direction of $\boldsymbol{r}$. See Appendix B for $G_{2 k+l}$. To make the text compact, we also put some other symbols and definitions there without further mention. We discuss a relationship between the LPG and the GWP in the next section.

It would be very interesting if the LPG could be extended to functions describing an $N$-particle system. Its possible candidate, a correlated Gaussian (CG), was actually proposed more than 20 years ago by $\mathrm{K}$. Varga and one of the present authors (Y.S.) $[4,5]$ by extending the spherical CG $[6,7]$ to that including a rotational motion of the system. The CG is concisely expressed as

$$
f_{K L M}^{u A}(\boldsymbol{\rho})=\mathcal{N}_{K L}^{u A}|\widetilde{u} \rho|^{2 K+L} Y_{L M}(\widehat{u} \boldsymbol{\rho}) e^{-\frac{1}{2} \widetilde{\rho} A \rho},
$$

where the column vector $\rho$ comprises $N-1$ relative coordinates, $\left(\boldsymbol{\rho}_{1}, \ldots, \boldsymbol{\rho}_{N-1}\right)$. The CG is characterized by a column vector $u=\left(u_{i}\right)$ of dimension $(N-1)$ and a symmetric, positive-definite $(N-1) \times(N-1)$ matrix $A=\left(A_{i j}\right)$. The symbol $\sim$ indicates a transpose of a column vector or a matrix, and therefore $\tilde{u} \rho$ (the so-called global vector) and $\widetilde{\rho} A \rho$ are shorthand notations for

$$
\tilde{u} \boldsymbol{\rho}=\sum_{i=1}^{N-1} u_{i} \boldsymbol{\rho}_{i}, \quad \tilde{\boldsymbol{\rho}} A \boldsymbol{\rho}=\sum_{i, j=1}^{N-1} A_{i j} \boldsymbol{\rho}_{i} \cdot \boldsymbol{\rho}_{j} .
$$

$K$ in Eq. (3) is a non-negative integer and $\mathcal{N}_{K L}^{u A}$ is a normalization constant (see also Ref. [8]). A formal resemblance of Eqs. (2) and (3) is apparent.

The purpose of this paper is to demonstrate that a linearchain (LC) state comprising the GWPs arranged in a row can in fact be very well represented by the CG with a suitable choice of $K, A$, and $u$, and furthermore to apply to the case of four $\alpha$ particles in order to examine whether the LC state can exist or not in ${ }^{16} \mathrm{O}$. Although it has been successful in a number of few-body problems [9-15], application of the CG has focused most on structures described well with small $K$ values, e.g., 0,1 , and 2 . We will open up a new application of the CG by compactly describing a strongly deformed state rotating with high angular momentum.

The LC structure in nuclei was proposed as a candidate for a strongly deformed state that may play an important role for some excited states, especially in light nuclei [16]. An experimental search was done in ${ }^{16} \mathrm{O}$ [17] but no firm confirmation has been made yet. A theoretical analysis of the decay scheme of the LC state was first made in Ref. [18]. The $4 \alpha$ decay of some excited states in ${ }^{16} \mathrm{O}$ has been studied experimentally [19-21]. Recently the possibility of nuclear LC states in ${ }^{16} \mathrm{O}$ as well as in other light nuclei has attracted renewed interest both theoretically and experimentally [22-32].

In Sec. II we first begin with an examination of the GWP with respect to the angular-momentum content, and show that the LPG can be a very convenient and flexible sp orbit representing a spatial localization. In Sec. III we prove that the $\mathrm{CG}$, an extension of the LPG to many-particle functions, is versatile enough to simulate a strongly deformed 
LC configuration of $N$ particles. We give a simple prescription for determining the $\mathrm{CG}$ parameters to fit the LC configuration as accurately as possible. An application of the present formulation is worked out in Sec. IV to examine possible LC states in ${ }^{16} \mathrm{O}$. The energy of the LC configuration of four $\alpha$ particles is studied by changing its size or length of the system as well as the total orbital angular momentum. Conclusions are drawn in Sec. V.

\section{SPATIALLY LOCALIZED SINGLE-PARTICLE ORBITS}

\section{A. Angular-momentum expansion of Gaussian wave packet}

The GWP (1) contains many partial waves. Its orbital angular-momentum content is analyzed as

$$
\phi_{s}^{v}(\boldsymbol{r})=\sum_{l m} \sqrt{4 \pi} b_{l}(\eta) \phi_{s l m}^{v}(\boldsymbol{r}) Y_{l m}^{*}(\hat{\boldsymbol{s}}),
$$

where $\phi_{s l m}^{v}(\boldsymbol{r})$ is a normalized shifted Gaussian (SG),

$$
\phi_{s l m}^{v}(\boldsymbol{r})=\frac{2 e^{-\eta}}{b_{l}(\eta)}\left(\frac{v^{3}}{\pi}\right)^{\frac{1}{4}} i_{l}(v s r) e^{-\frac{v}{2} r^{2}} Y_{l m}(\hat{\boldsymbol{r}})
$$

expressed in terms of the modified spherical Bessel function of the first kind [33], $i_{l}(x)=\sqrt{\frac{\pi}{2 x}} I_{l+\frac{1}{2}}(x)$. A dimensionless quantity $\eta$,

$$
\eta=\frac{1}{2} v s^{2}
$$

is a measure of the spatial localization of the packet.

The probability of finding the component with angular momentum $l$ in the GWP is defined by

$$
\begin{aligned}
P_{\mathrm{SG}}(l ; \eta) & =\sum_{m=-l}^{l} \frac{1}{4 \pi} \int d \hat{\boldsymbol{s}} 4 \pi\left[b_{l}(\eta)\right]^{2}\left|Y_{l m}(\hat{\boldsymbol{s}})\right|^{2} \\
& =(2 l+1)\left[b_{l}(\eta)\right]^{2},
\end{aligned}
$$

which satisfies a sum rule, $\sum_{l=0}^{\infty} P_{\mathrm{SG}}(l ; \eta)=1$. Figure 1 plots $P_{\mathrm{SG}}(l ; \eta)$ as a function of $l$ for some values of $\eta$. If $s$ is of the order of the nuclear surface $r_{0} A^{1 / 3}\left(r_{0}=1.1 \mathrm{fm}, A\right.$ is the mass number), $\eta$ varies as $0.58 A^{1 / 3}$ for the harmonic-oscillator (HO) choice of $v \approx 0.965 A^{-1 / 3} \mathrm{fm}^{-2}$. For example, $\eta$ is about 1.5 for $A=16$ and 3.4 for $A=208$, respectively. On the other hand, if an $\alpha$ cluster described with its sp $v$ value

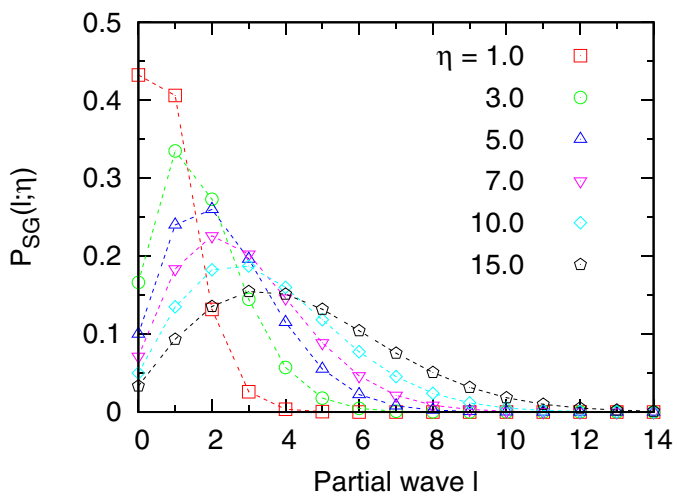

FIG. 1. Probability of finding the component with partial wave $l$ in the Gaussian wave packet characterized by $\eta$. See Eq. (8). $\left(0.521 \mathrm{fm}^{-2}\right)$ is localized at $s=8 \mathrm{fm}$ beyond the surface of ${ }^{208} \mathrm{~Pb}, \eta$ increases to about 17 , indicating the enhanced spatial localization. As shown in Fig. 1, the probability distribution extends to larger $l$ with increasing $\eta$, a consequence of the uncertainty relation between the angular momentum and the angular position. Many localized orbits with large $l$ are needed to represent the surface $\alpha$ clustering in the ${ }^{208} \mathrm{~Pb}$ region [34-36].

Including spatially correlated configurations in the $\mathrm{HO}$ shell-model description is very tough because they require many major-shell excitations. Because of this, even a largescale shell-model calculation is not able to reproduce some cluster states in light nuclei [37,38]. It is therefore important to develop simple sp orbits that are needed to construct such cluster states. Although a localized orbit like the SG could be a useful sp orbit, that is not a practically convenient basis function because calculations of various matrix elements are in general fairly involved. The LPG can instead be an ideal substitute, as shown in the next subsection.

\section{B. Locally peaked Gaussian}

We show that the LPG (2) well approximates the SG if $k$ and $a$ are appropriately chosen. The LPG has a merit that calculating matrix elements is easy. For example, the overlap between the LPG and the SG reads

$$
\left\langle\phi_{s l m}^{v} \mid \varphi_{k l m}^{a}\right\rangle=O_{l}(v s, a k) e^{-\frac{a \eta}{v+a}} F_{1}\left(-k, \bar{l}+1 ;-\frac{v \eta}{v+a}\right),
$$

where $\bar{l}$ stands for $l+1 / 2$, and ${ }_{1} F_{1}$ is the confluent hypergeometric function [33], which reduces to a polynomial for a non-negative integer $k$. To determine $k$ and $a$ that approximate a given SG as closely as possible, we require the expectation values of $r^{2}$ and $-\Delta$ calculated with the LPG,

$$
\begin{aligned}
\left\langle\varphi_{k l m}^{a}\left|r^{2}\right| \varphi_{k l m}^{a}\right\rangle & =(2 k+\bar{l}+1) \frac{1}{a}, \\
\left\langle\varphi_{k l m}^{a}|-\Delta| \varphi_{k l m}^{a}\right\rangle & =\left(1+\frac{\bar{l}^{2}}{2 k+\bar{l}}\right) a,
\end{aligned}
$$

to be equal to the corresponding values of the $\mathrm{SG}$,

$$
\begin{aligned}
\left\langle\phi_{s l m}^{v}\left|r^{2}\right| \phi_{s l m}^{v}\right\rangle & =\left(\bar{l}+1+\eta+\eta \frac{i_{l+1}(\eta)}{i_{l}(\eta)}\right) \frac{1}{v}, \\
\left\langle\phi_{s l m}^{v}|-\Delta| \phi_{s l m}^{v}\right\rangle & =\left(\bar{l}+1-\eta+\eta \frac{i_{l+1}(\eta)}{i_{l}(\eta)}\right) v .
\end{aligned}
$$

This requirement is natural because the SG is characterized by its peak position and the falloff of the peak height. The condition leads to $k$ and $a$ as follows:

$$
k=\frac{1}{2}(z-\bar{l}), \quad a=\frac{z+1}{\left\langle\phi_{s l m}^{v}\left|r^{2}\right| \phi_{s l m}^{v}\right\rangle},
$$

where $z=\frac{1}{2}(b+\sqrt{D})$, with $b$ and $D$ being given by

$$
\begin{aligned}
b & =\left(\bar{l}+1+\eta \frac{i_{l+1}(\eta)}{i_{l}(\eta)}\right)^{2}-\bar{l}^{2}-1-\eta^{2}, \\
D & =b^{2}-4 \bar{l}^{2} .
\end{aligned}
$$




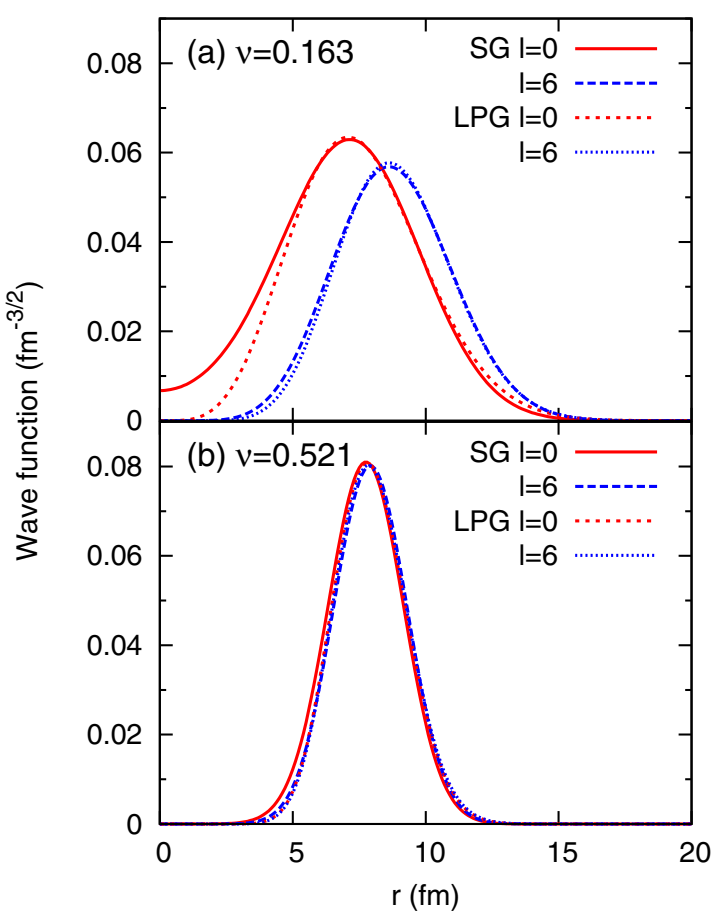

FIG. 2. Comparison of the radial functions with $l=0$ and 6 between the SG, $\phi_{s l}^{v}(r)$, and the LPG, $\varphi_{k l}^{a}(r)$. The value of $s$ is set to $8 \mathrm{fm}$. Both $v$ and $a$ are given in $\mathrm{fm}^{-2}$. Case (a) $v=0.163$ : $(a, k)=(0.0796,2)$ for $l=0$ and $(0.1076,1)$ for $l=6$. Case $(b)$ $v=0.521:(a, k)=(0.2630,8)$ for $l=0$ and $(0.2605,5)$ for $l=6$. The overlap integral between the LPG and the SG is larger than 0.998 in all the cases.

We have numerically checked that $b-2 \bar{l}$ is non-negative, which guarantees that $k$ is non-negative. See Appendix $C$ for this. For a practical purpose, $k$ is restricted to a non-negative integer closest to $(z-\bar{l}) / 2$. Once $k$ is fixed, $a$ is set to maximize the overlap (9) between the arithmetic and geometric means of two $a$ values that reproduce the respective expectation values of $r^{2}$ and $-\Delta$ of the SG. Figure 2 compares $l=0$ and 6 radial functions between the LPG and the SG with $s=8 \mathrm{fm}$. In case (a), $v=0.163 \mathrm{fm}^{-2}$ is the $\mathrm{HO}$ size parameter appropriate in the ${ }^{208} \mathrm{~Pb}$ region, whereas $v=0.521 \mathrm{fm}^{-2}$ in case (b) reproduces the size of the $\alpha$ particle with the $(0 s)^{4}$ configuration. $\eta$ is quite different: 5.2 in case (a) and 16.7 in case (b). In both cases the LPG very well approximates the SG.

\section{LINEAR-CHAIN CONFIGURATIONS OF $N$ PARTICLES}

\section{A. Gaussian wave-packet representation}

For the sake of simplicity, we assume that $N$ particles have an equal mass $m$. In the LC state they are conveniently described by the GWPs that are all centered at some positions on a straight line passing through the coordinate origin. This intrinsic state rotates around the center of mass (c.m.) of the system with high angular velocity. Let a unit vector $\boldsymbol{e}(|\boldsymbol{e}|=1)$ denote the direction of the line. The LC state with the total orbital angular momentum $L$ and its projection $M$ is defined by

$$
\Phi_{L M}=\mathcal{N}_{L} \int d \boldsymbol{e} Y_{L M}(\hat{\boldsymbol{e}}) \prod_{i=1}^{N} \phi_{S_{i} \boldsymbol{e}}^{v}\left(\boldsymbol{r}_{i}\right) .
$$

The $i$ th particle is centered at the position $S_{i} \boldsymbol{e}$ on the line. We assume that $\sum_{i=1}^{N} S_{i}=0$ to ensure that the c.m. motion of the system remains fixed around the origin. $\mathcal{N}_{L}$ is a normalization constant given by $\mathcal{N}_{L}=1 / \sqrt{4 \pi} b_{L}(H)$, where $H$ is an analog of the localization measure $\eta(7)$ :

$$
H=\frac{1}{2} \nu \sum_{i=1}^{N} S_{i}^{2}=\frac{1}{2} \nu \widetilde{S} S .
$$

Here $S=\left(S_{i}\right)$ is an $N$-dimensional column vector.

The c.m. motion of $\Phi_{L M}$ is separated by transforming the sp coordinates $\boldsymbol{r}=\left(\boldsymbol{r}_{i}\right)$ to a set of the relative coordinates, $\boldsymbol{\rho}=\left(\boldsymbol{\rho}_{i}\right)$, and the c.m. coordinate $\boldsymbol{R}_{N}$ :

$$
\left(\begin{array}{c}
\rho \\
\boldsymbol{R}_{N}
\end{array}\right)=U \boldsymbol{r}, \quad \boldsymbol{r}=U^{-1}\left(\begin{array}{c}
\rho \\
\boldsymbol{R}_{N}
\end{array}\right) .
$$

If $\rho$ is a set of Jacobi coordinates, $U$ and $U^{-1}$ are

$$
\begin{aligned}
U & =\left(\begin{array}{ccccc}
-1 & 1 & 0 & \cdots & 0 \\
-\frac{1}{2} & -\frac{1}{2} & 1 & \cdots & 0 \\
\vdots & & & & \vdots \\
-\frac{1}{N-1} & -\frac{1}{N-1} & \cdots & \cdots & 1 \\
\frac{1}{N} & \frac{1}{N} & \cdots & \cdots & \frac{1}{N}
\end{array}\right), \\
U^{-1} & =\left(\begin{array}{ccccc}
-\frac{1}{2} & -\frac{1}{3} & \cdots & -\frac{1}{N} & 1 \\
\frac{1}{2} & -\frac{1}{3} & \cdots & -\frac{1}{N} & 1 \\
\vdots & & & & \vdots \\
0 & 0 & \cdots & \cdots & 1 \\
0 & 0 & \cdots & \frac{N-1}{N} & 1
\end{array}\right) .
\end{aligned}
$$

Let $U_{J}$ denote the first $(N-1) \times N$ submatrix of $U$ and $U_{J}^{-1}$ the first $N \times(N-1)$ submatrix of $U^{-1}$. Note the following identities:

$$
\begin{aligned}
U_{J} \widetilde{U_{J}} & =\Lambda^{-1}, \widetilde{U_{J}^{-1}} U_{J}^{-1}=\Lambda, \\
U_{J}^{-1} \Lambda^{-1} \widetilde{U_{J}^{-1}} & =U_{J}^{-1} U_{J}=1_{N}-\frac{1}{N} E_{N},
\end{aligned}
$$

where $1_{N}$ is the $N \times N$ unit matrix, $E_{N}$ is the $N \times N$ matrix unit whose elements are all unity, and $\Lambda$ is an $(N-1) \times$ $(N-1)$ diagonal matrix defined by

$$
\Lambda_{i j}=\frac{i}{i+1} \delta_{i, j} .
$$

The LC state (14) reduces to a product of the c.m. part and the normalized intrinsic part $\Phi_{S L M}^{v}(\mathrm{LC})$ :

$$
\Phi_{L M}=\phi_{\mathbf{0}}^{N v}\left(\boldsymbol{R}_{N}\right) \Phi_{S L M}^{v}(\mathrm{LC})
$$

where

$$
\begin{aligned}
& \Phi_{S L M}^{v}(\mathrm{LC}) \\
& =\frac{2 e^{-H}}{b_{L}(H)}\left(\frac{v^{3 N-3}}{N^{3} \pi^{3 N-5}}\right)^{\frac{1}{4}} e^{-\frac{1}{2} \widetilde{\rho} A_{0} \rho} i_{L}\left(\left|\widetilde{u_{0}} \boldsymbol{\rho}\right|\right) Y_{L M}\left(\widetilde{u_{0} \boldsymbol{\rho}}\right)
\end{aligned}
$$


with

$$
A_{0}=v \Lambda, \quad u_{0}=v \widetilde{U_{J}^{-1}} S
$$

Note that the parity of $\Phi_{S L M}^{v}(\mathrm{LC})$ is $(-1)^{L}$.

Some basic operators are conveniently expressed in terms of $\rho$. For example,

$$
\begin{aligned}
\boldsymbol{r}_{i}-\boldsymbol{R}_{N} & =\sum_{k=1}^{N-1}\left(U_{J}^{-1}\right)_{i k} \boldsymbol{\rho}_{k}, \\
\boldsymbol{r}_{i}-\boldsymbol{r}_{j} & =\sum_{k=1}^{N-1} \omega_{k}^{(i j)} \boldsymbol{\rho}_{k}=\widetilde{\omega^{(i j)} \boldsymbol{\rho}}
\end{aligned}
$$

with

$$
\omega_{k}^{(i j)}=\left(U_{J}^{-1}\right)_{i k}-\left(U_{J}^{-1}\right)_{j k} .
$$

The hyperradius $R$ of the system is defined by

$$
R^{2}=\sum_{i=1}^{N}\left(\boldsymbol{r}_{i}-\boldsymbol{R}_{N}\right)^{2}=\sum_{i=1}^{N-1} \frac{i}{i+1} \boldsymbol{\rho}_{i}^{2}=\tilde{\boldsymbol{\rho}} \Lambda \boldsymbol{\rho} .
$$

The kinetic energy with the c.m. kinetic-energy $T_{\text {c.m. }}$ being subtracted reads

$$
T_{\text {in }}=\sum_{i=1}^{N} \frac{\boldsymbol{p}_{i}^{2}}{2 m}-T_{\text {c.m. }}=\sum_{i=1}^{N-1} \frac{i+1}{2 m i} \boldsymbol{\pi}_{i}^{2}=\frac{1}{2 m} \tilde{\boldsymbol{\pi}} \Lambda^{-1} \boldsymbol{\pi},
$$

where $\boldsymbol{\pi}=\left(\boldsymbol{\pi}_{j}\right)$ with $\boldsymbol{\pi}_{j}=-i \hbar \partial / \partial \boldsymbol{\rho}_{j}$ is a column vector of dimension $(N-1)$.

The LC state (21) takes a form quite similar to the SG (6). The argument of $i_{l}(v s r)$ becomes $v s^{2}=2 \eta$ at the peak of the SG. Likewise, since $\Phi_{L M}$ is peaked at $\boldsymbol{r}=S \boldsymbol{e}, \Phi_{S L M}^{v}(\mathrm{LC})$ is peaked at $\boldsymbol{\rho}=U_{J} S \boldsymbol{e}$. For this $\boldsymbol{\rho}$, the argument of $i_{L}\left(\left|\widetilde{u}_{0} \rho\right|\right)$ becomes $\left|\widetilde{u_{0}} \rho\right|=\nu \widetilde{S} S|\boldsymbol{e}|=2 H$. Similarly to the sp SG case, $H$ is indeed the localization measure for the $N$-body LC state. This analogy becomes more substantial by calculating the expectation values of $R^{2}$ and $T_{\text {in }}$ [cf. Eq. (11)]:

$$
\begin{aligned}
& \left\langle\Phi_{S L M}^{v}(\mathrm{LC})\left|R^{2}\right| \Phi_{S L M}^{v}(\mathrm{LC})\right\rangle \\
& \quad=\frac{1}{v}\left[\frac{3}{2}(N-1)+L+H+H \frac{i_{L+1}(H)}{i_{L}(H)}\right], \\
& \left\langle\Phi_{S L M}^{v}(\mathrm{LC})\left|T_{\text {in }}\right| \Phi_{S L M}^{v}(\mathrm{LC})\right\rangle \\
& \quad=\frac{\hbar^{2} v}{2 m}\left[\frac{3}{2}(N-1)+L-H+H \frac{i_{L+1}(H)}{i_{L}(H)}\right] .
\end{aligned}
$$

This kinetic-energy expectation value gives approximate $L(L+1)$ dependence up to large $L$ already for $H \geqslant 30$ in spite of its opacity. See Appendix $C$ for some details.

\section{B. Correlated-Gaussian approximation}

We have shown that in the one-variable case the LPG (2) very well approximates the SG (6) and also that the functional form of the $N$-particle LC state (21) is similar to that of the SG. Here we show that the LC state can be very well approximated by the CG (3), which is a natural extension of the LPG to the many-variable case.
Various matrix elements with the CG can easily be obtained by making use of its generating function $g$ [4]:

$$
\begin{aligned}
f_{K L M}^{u A}(\boldsymbol{\rho})= & \frac{\mathcal{N}_{K L}^{u A}}{B_{K L}} \int d \boldsymbol{e} Y_{L M}(\hat{\boldsymbol{e}}) \\
& \times\left(\frac{d^{2 K+L}}{d \alpha^{2 K+L}} g(\alpha, \boldsymbol{e} ; u, A, \boldsymbol{\rho})\right)_{\alpha=0},
\end{aligned}
$$

where $\boldsymbol{e}$ is a unit vector. The overlap of Eqs. (21) and (3) is (see Ref. [8] for details)

$$
\begin{aligned}
& \left\langle f_{K L M}^{u A} \mid \Phi_{S L M}^{v}(\mathrm{LC})\right\rangle \\
& \quad=\frac{e^{-H}}{b_{L}(H)}\left(\frac{\operatorname{det} 4 v A}{N(\operatorname{det} B)^{2}}\right)^{\frac{3}{4}} \frac{e^{p_{0}}}{\sqrt{\bar{p}^{2 K+L}}} G_{K L}(p, q),
\end{aligned}
$$

where the matrix $B$ is $B=A+A_{0}$ and

$$
\begin{aligned}
& \bar{p}=\frac{1}{4} \widetilde{u} A^{-1} u, \quad p=\frac{1}{2} \widetilde{u} B^{-1} u, \\
& q=\tilde{u} B^{-1} u_{0}, \quad p_{0}=\frac{1}{2} \widetilde{u_{0}} B^{-1} u_{0} .
\end{aligned}
$$

To determine the CG parameters, $(A, u, K)$, that well approximate $\Phi_{S L M}^{v}(\mathrm{LC})$ characterized by $(\nu, S)$ or $\left(A_{0}, u_{0}\right)$, we follow the same route as in Sec. II B. The expectation values (28) are compared to those with the CG (3) [8]:

$$
\begin{aligned}
& \left\langle f_{K L M}^{u A}\left|R^{2}\right| f_{K L M}^{u A}\right\rangle \\
& \quad=\frac{3}{2} \operatorname{Tr} A^{-1} \Lambda+(L+2 K) \frac{\bar{q}}{\bar{p}}, \\
& \left\langle f_{K L M}^{u A}\left|T_{\text {in }}\right| f_{K L M}^{u A}\right\rangle \\
& \quad=\frac{\hbar^{2}}{2 m}\left[\frac{3}{2} \operatorname{Tr} A \Lambda^{-1}+\left(L-2 K+4 C_{K L}\right) \frac{\bar{\lambda}}{\bar{p}}\right],
\end{aligned}
$$

where

$$
\begin{aligned}
\bar{q} & =\frac{1}{4} \widetilde{u} A^{-1} \Lambda A^{-1} u, \quad \bar{\lambda}=\frac{1}{4} \widetilde{u} \Lambda^{-1} u, \\
C_{K L} & =\frac{1}{\gamma_{K K L}(1)} \gamma_{K K L}^{\prime}(1) .
\end{aligned}
$$

Here $\gamma_{K K^{\prime} L}^{\prime}(x)=\frac{d}{d x} \gamma_{K K^{\prime} L}(x)$. As a simplest choice, let us assume that $A$ is proportional to $A_{0}$ :

$$
A=a A_{0}=a \nu \Lambda \text {. }
$$

The condition to be satisfied then reads

$$
\begin{aligned}
& \frac{1}{a} {\left[\frac{3}{2}(N-1)+L+2 K\right] } \\
& \quad=\frac{3}{2}(N-1)+L+H+H \frac{l_{L+1}(H)}{i_{L}(H)}, \\
& a\left[\frac{3}{2}(N-1)+L-2 K+4 C_{K L}\right] \\
& \quad=\frac{3}{2}(N-1)+L-H+H \frac{i_{L+1}(H)}{i_{L}(H)} .
\end{aligned}
$$

$K$ is determined by requiring the product of the left-hand sides of Eq. (35), which is a function of $K$ and independent of $a$, to be equal to that of the right-hand sides. Since $K$ is set to a non-negative integer, the condition may not be perfectly met, 
TABLE I. Maximum overlap, $\left\langle\Phi_{S L M}^{v}(\mathrm{LC}) \mid f_{S L M}^{v}(\mathrm{LC}: \mathrm{CG})\right\rangle$, between the CG and the LC states with the localization measure $H$ for a system of four particles $(N=4) . K$ and $a$ (or $A$ ) are the CG parameters that maximize the overlap with the LC state.

\begin{tabular}{|c|c|c|c|c|c|c|c|c|c|c|c|c|c|c|c|}
\hline$L \backslash H$ & & 10 & 20 & 30 & 40 & 50 & 60 & 70 & 80 & 90 & 100 & 140 & 180 & 220 & 260 \\
\hline \multirow[t]{2}{*}{0} & $K$ & 8 & 17 & 25 & 34 & 43 & 52 & 60 & 69 & 78 & 87 & 122 & 157 & 192 & 227 \\
\hline & $a$ & 0.872 & 0.879 & 0.866 & 0.871 & 0.874 & 0.877 & 0.871 & 0.873 & 0.875 & 0.876 & 0.876 & 0.876 & 0.875 & 0.875 \\
\hline \multirow[t]{2}{*}{10} & $K$ & 6 & 13 & 22 & 30 & 39 & 47 & 56 & 65 & 73 & 82 & 117 & 152 & 187 & 222 \\
\hline & $a$ & 0.933 & 0.880 & 0.891 & 0.878 & 0.882 & 0.874 & 0.876 & 0.878 & 0.873 & 0.875 & 0.875 & 0.875 & 0.875 & 0.875 \\
\hline \multirow{2}{*}{20} & $a$ & 0.940 & 0.924 & 0.893 & 0.887 & 0.879 & 0.885 & 0.878 & 0.881 & 0.875 & 0.878 & 0.878 & 0.874 & 0.875 & 0.875 \\
\hline & Overlap & 0.993 & 0.985 & 0.981 & 0.978 & 0.977 & 0.976 & 0.976 & 0.975 & 0.975 & 0.975 & 0.975 & 0.974 & 0.974 & 0.974 \\
\hline \multirow[t]{3}{*}{30} & $K$ & 5 & 11 & 18 & 25 & 33 & 41 & 49 & 57 & 66 & 74 & 109 & 143 & 178 & 213 \\
\hline & $a$ & 0.966 & 0.936 & 0.920 & 0.899 & 0.895 & 0.890 & 0.884 & 0.879 & 0.883 & 0.878 & 0.880 & 0.876 & 0.876 & 0.876 \\
\hline & Overlap & 0.996 & 0.990 & 0.985 & 0.982 & 0.980 & 0.978 & 0.977 & 0.977 & 0.976 & 0.976 & 0.975 & 0.975 & 0.975 & 0.975 \\
\hline
\end{tabular}

but $K$ is fixed so as to satisfy the condition as much as possible. For this $K$ we have two $a$ values: one determined from the first equation in Eq. (35) and the other determined from the second equation. The values are found to be almost equal, and we choose $a$ as an arithmetic average of those two values. Note that $K$ and $a$ or $A$ are determined depending on $L, H$, and $N$ but independent of $u$.

Once $A$ and $K$ are set, the overlap (30) depends on $u$ only through the term

$$
\frac{1}{\sqrt{\bar{p}^{2 K+L}}} G_{K L}(p, q)=\left(\frac{2 a}{a+1}\right)^{\frac{1}{2}(2 K+L)} G_{K L}(1, z)
$$

with

$$
z=\sqrt{\frac{2}{(a+1) v}} \frac{\tilde{u} \Lambda^{-1} u_{0}}{\sqrt{\tilde{u} \Lambda^{-1} u}} .
$$

The overlap becomes a maximum when $G_{K L}(1, z)$ or $z$ reaches a maximum. The maximum of $z$ occurs for such $u$ that is proportional to $u_{0}$, i.e., $\max (z)=\sqrt{4 H /(a+1)}$. For definiteness, $u$ is set equal to $u_{0}$. In this way the CG that has the maximum overlap with $\Phi_{S L M}^{v}(\mathrm{LC})$ is determined to be $f_{K L M}^{u_{0} a A_{0}}(\boldsymbol{\rho})$, which is denoted $f_{S L M}^{v}(\mathrm{LC}: \mathrm{CG})$ in order to emphasize its LC character. As shown in Eq. (22), $A_{0}$ is unique but $u_{0}$ depends on the column vector $S$ for a given $H$. The CGs with different $u_{0}$ parameters all have the same maximum overlap with $\Phi_{S L M}^{v}(\mathrm{LC})$. Table I lists the CG parameters, $K$ and $a$, determined in this way together with the maximum overlap, $\left\langle\Phi_{S L M}^{v}(\mathrm{LC}) \mid f_{S L M}^{v}(\mathrm{LC}: \mathrm{CG})\right\rangle$ for some sets of $H$ and $L$ values. Observing that it is close to unity, we conclude that the LC configuration can be well approximated with the CG (3) provided its parameters are determined as mentioned above. It is worth stressing that the CG approximation works excellently even for extremely large $L$. Numerical angular momentum projection for such $L$ states may be tough in general. No such difficulty arises here thanks to the analytic manipulation.
The overlap of two CGs has a simple dependence on their parameters:

$$
\begin{aligned}
\left\langle f_{K L M}^{u A} \mid f_{K^{\prime} L M}^{v B}\right\rangle= & \frac{\gamma_{K K^{\prime} L}(\bar{t}) \bar{t}^{\frac{L}{2}}}{\sqrt{\gamma_{K K L}(1) \gamma_{K^{\prime} K^{\prime} L}(1)}} \\
& \times\left(\frac{\sqrt{\operatorname{det} A B}}{\operatorname{det} C}\right)^{\frac{3}{2}}\left(\frac{\widetilde{u} C^{-1} u}{\widetilde{u} A^{-1} u}\right)^{K}\left(\frac{\widetilde{v} C^{-1} v}{\widetilde{v} B^{-1} v}\right)^{K^{\prime}},
\end{aligned}
$$

where

$$
C=\frac{1}{2}(A+B), \quad \bar{t}=\frac{\left(\widetilde{u} C^{-1} v\right)^{2}}{\left(\widetilde{u} A^{-1} u\right)\left(\widetilde{v} B^{-1} v\right)} .
$$

As shown in the table, $f_{S L M}^{\nu}(\mathrm{LC}: \mathrm{CG})$ may have very large $K$. On the other hand, low-lying states are described well with the CGs with small or even $K=0$ values. A specific overlap, $\left\langle f_{K L M}^{u A} \mid f_{0 L M}^{u A}\right\rangle$, reduces to $1 / \sqrt{\gamma_{K K L}(1)}$, and becomes very small for very large $K$.

\section{Geometrical shape and deformation}

Angular-momentum projection is often carried out after a variational calculation is first performed by using unprojected basis functions. This is the so-called variation before projection (VBP). It is of course desirable to perform the projection before variation, that is, the variation after projection (VAP). Since the angular-momentum projection usually takes expensive computer-time in the numerical integration over the Euler angles (see, e.g., Ref. [39]), the VBP is employed in most calculations. The angular-momentum projection is often not performed fully but is treated in a cranking model approximation [40-42]. The CG already carries good angular momentum, making the VAP calculation very easy.

In the VBP calculation, the geometrical shape of the unprojected configuration is often discussed. Although such shape is not "observable," the VBP basis functions give an intuitive image of the state, as shown in, e.g., Refs. [22-27]. An elegant way to extract the intrinsic density from the VAP wave function was shown in Ref. [43]. Here we discuss a simpler way to get the geometrical picture from the CG. To 
characterize the intrinsic shape, we can make use of a set of operators, $r_{i j}^{2}$ and $\left(\boldsymbol{r}_{j}-\boldsymbol{r}_{i}\right) \cdot\left(\boldsymbol{r}_{k}-\boldsymbol{r}_{j}\right)(i<j<k)$, for all pairs, and let $D_{i j}^{2}$ and $D_{i j} D_{j k} \cos \Theta_{i j k}$ denote their expectation values. All sets of $D_{i j}$ and $\Theta_{i j k}$ serve to extract the shape. Note that those operators are all expressed in a concise form, $\tilde{\rho} \Omega \rho$, with the $(N-1) \times(N-1)$ matrix $\Omega$ being $\omega^{(i j)} \widetilde{\omega^{(i j)}}$ and $\omega^{(j i)} \widetilde{\omega^{(k j)}}$, respectively. See Eq. (24). The matrix element of $\tilde{\rho} \Omega \rho$ is obtained in exactly the same way as that of $R^{2}$. We give it in Appendix D for convenience.

To get information on the deformation of the VAP wave function $\Psi_{L M}$, we may use the matrix element of the (mass) quadrupole moment $Q$,

$$
\left\langle\Psi_{L L}|Q| \Psi_{L L}\right\rangle
$$

where

$$
Q=3 R_{z}^{2}-R^{2}
$$

Here $R_{z}^{2}=\sum_{i=1}^{N}\left(z_{i}-Z_{N}\right)^{2}$ with $z_{i}-Z_{N}$ being the $z$ component of $\boldsymbol{r}_{i}-\boldsymbol{R}_{N}$. We define a quantity $\delta_{2}$,

$$
\delta_{2}=\frac{\left\langle\Psi_{L L}|Q| \Psi_{L L}\right\rangle}{\left\langle\Psi_{L M}\left|R^{2}\right| \Psi_{L M}\right\rangle},
$$

which leads to the following relation:

$$
\frac{\left\langle x^{2}\right\rangle+\left\langle y^{2}\right\rangle}{\left\langle z^{2}\right\rangle}=\frac{2-\delta_{2}}{1+\delta_{2}},
$$

where, e.g., $\left\langle z^{2}\right\rangle$ stands for $\left\langle\Psi_{L L}\left|R_{z}^{2}\right| \Psi_{L L}\right\rangle$, and we discuss the deformation of the LC state in Sec. IV D. Appendix E gives a formula to calculate the matrix element $\left\langle f_{K L L}^{u A}|Q| f_{K^{\prime} L L}^{v B}\right\rangle$.

\section{FOUR- $\alpha$ LINEAR-CHAIN STATES}

\section{A. Potential parameters}

A two-body $\alpha-\alpha$ potential we use here is the same as that used in Ref. [44]. It consists of nuclear $\left(V_{2 B}\right)$ and Coulomb $\left(V_{\mathrm{C}}\right)$ terms:

$$
\begin{aligned}
v_{i j}= & 125 \exp \left(-\frac{r_{i j}^{2}}{1.53^{2}}\right)-30.18 \exp \left(-\frac{r_{i j}^{2}}{2.85^{2}}\right) \\
& +\frac{4 e^{2}}{r_{i j}} \operatorname{erf}\left(0.60141 r_{i j}\right) .
\end{aligned}
$$

Energy and length are given in units of $\mathrm{MeV}$ and $\mathrm{fm}$, respectively. A three- $\alpha$ nuclear potential, $V_{3 \mathrm{~B}}=\sum_{i<j<k} v_{i j k}$, is also introduced. We may express it as

$$
v_{i j k}=v(i j) v(j k) v(k i)
$$

with

$$
v(i j)=v_{r} \exp \left(-\frac{r_{i j}^{2}}{\rho_{r}^{2}}\right)+v_{a} \exp \left(-\frac{r_{i j}^{2}}{\rho_{a}^{2}}\right) .
$$

$v(i j)$ is assumed to be $J^{\pi}$ independent. The parameters used in Ref. [44] are $v_{r}=0, v_{a}=-5.49 \mathrm{MeV}^{1 / 3}$, and $\rho_{a}=3.395$, if $v_{a}$ is replaced by the average of the $0^{+}$and $2^{+}$strengths. With the mass of the $\alpha$ particle and the charge constant, $\hbar^{2} / m=$ $10.5254 \mathrm{MeV} \mathrm{fm}^{2}, e^{2}=1.43996 \mathrm{MeV} \mathrm{fm}$, the energies of the $3 \alpha 0^{+}$ground state and the first excited $2^{+}$state calculated with that three-body potential are respectively about -10.9 and
$-1.6 \mathrm{MeV}$, which are compared to the experimental values of ${ }^{12} \mathrm{C},-7.28$ and $-2.84 \mathrm{MeV}$. These energies are obtained by using the CGs (3), in which the $2 \times 2$ matrix $A$ is provided with the ansatz

$$
\tilde{\boldsymbol{\rho}} A \boldsymbol{\rho}=\sum_{j>i=1}^{3} \frac{\left(\boldsymbol{r}_{i}-\boldsymbol{r}_{j}\right)^{2}}{b_{i j}^{2}}
$$

where $b_{i j}$ is chosen for each $i, j$ in a geometric progression as $b_{0} p^{n-1}\left(n=1, \ldots, N_{p}\right)$. Also nonzero $K$ values with $K \leqslant 2$ are allowed. The energy depends on the parameters $b_{0}, p$, and $N_{p}$ as well as $u$. A slight improvement is possible by including the short-ranged repulsive force with nonzero $v_{r}$, the existence of which is physically reasonable considering the Pauli principle acting between the $\alpha-\alpha$ relative motion [45]. With the parameters, $v_{r}=6.5 \mathrm{MeV}^{1 / 3}, \rho_{r}=1.43, v_{a}=$ $-6.0 \mathrm{MeV}^{1 / 3}$, and $\rho_{a}=3.40$, the $0^{+}$and $2^{+}$energies turn out to be about -8.7 and $-1.4 \mathrm{MeV}$. In what follows we use this three-body potential.

The width parameter $v$ of the GWP specifies the spatial extension of the c.m. motion of the $\alpha$ particle. It appears in the LC configuration (21), or its approximated CG. Since it is four times the sp HO parameter of the $\alpha$ particle, we set $v$ to $2.084 \mathrm{fm}^{-2}$. The energy we calculate is taken to be the one measured from the $4 \alpha$ threshold ( $E_{\mathrm{th}}=14.436 \mathrm{MeV}$ from the ground state of ${ }^{16} \mathrm{O}$ ).

\section{B. Features of correlated-Gaussian calculations}

Incorporating the boson symmetry of $\alpha$ particles in the $\mathrm{CG}$ formalism is very easy [4,5]. The permutation $P=\left(\begin{array}{cccc}1 & 2 & \cdots & N \\ P_{1} & P_{2} & \cdots & P_{N}\end{array}\right)$ changes $\boldsymbol{r}_{i} \rightarrow \boldsymbol{r}_{P_{i}}(i=1, \ldots, N)$, namely $\boldsymbol{r} \rightarrow \mathcal{P} \boldsymbol{r}$, with the $N \times N$ matrix $\mathcal{P}$ being defined by $\mathcal{P}_{i j}=\delta_{j, P_{i}}$. With this permutation, $\rho=U_{J} \boldsymbol{r}$ undergoes the transformation $\rho \rightarrow U_{J} \mathcal{P} \boldsymbol{r}$. Substitution of $\boldsymbol{r}=U_{J}^{-1} \rho$ proves that $P$ transforms $\rho$ to $U_{J} \mathcal{P} U_{J}^{-1} \rho \equiv T_{P} \rho$. Thus the CG (3) is subject to the following change: $P f_{K L M}^{u A}(\boldsymbol{\rho})=f_{K L M}^{\widetilde{T_{P}} u \widetilde{T_{P}} A T_{P}}(\boldsymbol{\rho})$; that is, the permutation $P$ sets the CG to a CG with $A$ and $u$ being replaced by $\widetilde{T_{P}} A T_{P}$ and $\widetilde{T_{P}} u$. In exactly the same way, a different choice of the relative coordinate set can be very easily incorporated in the $\mathrm{CG}$ formalism.

We evaluate the CG matrix elements using the formula given in Ref. [8]. To calculate the matrix element of the Gaussian-type potential we can use a much simpler route as follows. The use of Eq. (24) enables us to express $v_{i j}=e^{-a r_{i j}^{2}}$ as $e^{-a \tilde{\rho} \Omega^{(i j)} \rho}$ with $\Omega^{(i j)}=\omega^{(i j)} \widetilde{\omega^{(i j)}}$. The matrix element of the potential thus reduces to that of overlap type. The three-body force of Gaussian radial form is also treated in exactly the same way as the two-body case. The matrix element of the Coulomb potential is calculated by applying the above result. The Coulomb potential, $v_{C}(r)=\operatorname{erf}(\beta r) / r$, is expressed as an integral of the Gaussian-type potential

$$
v_{C}(r)=\frac{2 \beta}{\sqrt{\pi}} \int_{0}^{1} d z e^{-\beta^{2} z^{2} r^{2}},
$$

and we reduce its matrix element to that of the Gaussian-type potential with a variable range parameter $a=\beta^{2} z^{2}$. 


\section{Arrangements of four $\alpha$ particles}

Searching for $4 \alpha$ LC states requires a careful study of their energies with respect to the angular momentum and chain length. We examine the energy of the LC configuration with $J^{\pi}=L^{+}$(even $L$ ) by changing $H$ or equivalently the root-mean-square (rms) radius. It is important to get a global change of the system's energy with respect to that key parameter [46,47].

For a given $H$ there are different sets of $S$, denoted $S_{\kappa}^{H}$. Each $S_{\kappa}^{H}$ defines the LC configuration that is very well approximated by the CG as shown in Sec. III B. The set $S_{\kappa}^{H}$ corresponds to the vibration of $\alpha$ particles along the line of the LC state. Possible independent sets are prepared as follows. By eliminating one of the elements of $S$, say, $S_{4}=-\left(S_{1}+S_{2}+S_{3}\right), H$ reads

$$
\begin{aligned}
H & =v\left(S_{1}^{2}+S_{2}^{2}+S_{3}^{2}+S_{1} S_{2}+S_{1} S_{3}+S_{2} S_{3}\right) \\
& =v \widetilde{\varsigma} \mathcal{M} \varsigma,
\end{aligned}
$$

where $\varsigma$ is a column vector comprising the elements $S_{1}, S_{2}, S_{3}$ and $\mathcal{M}$ is a $3 \times 3$ matrix with $\mathcal{M}_{i i}=1, \mathcal{M}_{i \neq j}=1 / 2$. The eigenvalues of $\mathcal{M}$ are $2,1 / 2$, and $1 / 2$. With a suitable $3 \times 3$ orthogonal matrix $\mathcal{T}, \varsigma=\mathcal{T} Z, H$ can be recast to a quadratic form,

$$
H=v\left(2 Z_{1}^{2}+\frac{1}{2} Z_{2}^{2}+\frac{1}{2} Z_{3}^{2}\right) .
$$

By parametrizing $Z$ in terms of two angles, $\theta(0 \leqq \theta<$ $2 \pi)$ and $\phi(0 \leqq \phi<2 \pi)$, as $Z_{1}=\sqrt{H / 2 v} \cos \theta, \quad Z_{2}=$ $\sqrt{2 H / v} \sin \theta \cos \phi$, and $Z_{3}=\sqrt{2 H / v} \sin \theta \sin \phi$, we can cover all possible vectors $S$. We discretize $\theta$ and $\phi$ in a $5^{\circ}$ mesh to generate $S_{\kappa}^{H}$, and allow those configurations that have mutual overlaps of less than 0.85 with others in order to avoid possible linear dependence of the basis functions.

The total wave function for the LC state is in general given as a superposition of different LC configurations of four $\alpha$ particles:

$$
\Psi_{L M}=\sum_{H \kappa} C_{S_{\kappa}^{H}} \mathcal{S} f_{S_{\kappa}^{H} L M}^{v}(\mathrm{LC}: \mathrm{CG}),
$$

where the operator $\mathcal{S}=\sum_{P} P$ ensures extraction of a totally symmetric state.

\section{Search for linear-chain states}

First we compare the energies calculated with $f_{S_{\kappa}^{H} L M}^{\nu}(\mathrm{LC}: \mathrm{CG})$ by varying $S_{\kappa}^{H}$ for a given $H$. This is called a single $S$ (SS) model. The lowest energy found in this model is plotted in panel (a) of Fig. 3 as a function of $H$. The SS model finds a local energy minimum for $L=0,2,4,6,8$, and 10 , although the minima for $L=8,10$ are very shallow. The minima of $L=0,2,4$ appear at $H \approx 50$, which corresponds to the point $\alpha$ rms radius, $r_{\mathrm{rms}} \approx 3.52 \mathrm{fm}$. The minimum of the $L=6$ curve shifts to $H \approx 60$. For $L>12$, no energy minimum appears in the region of $H<130$, and the energy simply decreases with increasing $H$.

As discussed above, the energy of the SS model with $L=0$ becomes a minimum at $H \approx 50$, in which four $\alpha$ particles are positioned at $S_{1}=-4.295, S_{2}=-1.921, S_{3}=1.302$, $S_{4}=4.914 \mathrm{fm}$. This LC configuration is approximated by the $\mathrm{CG}$ that has $K=43$ and $a=0.874$. Before the boson

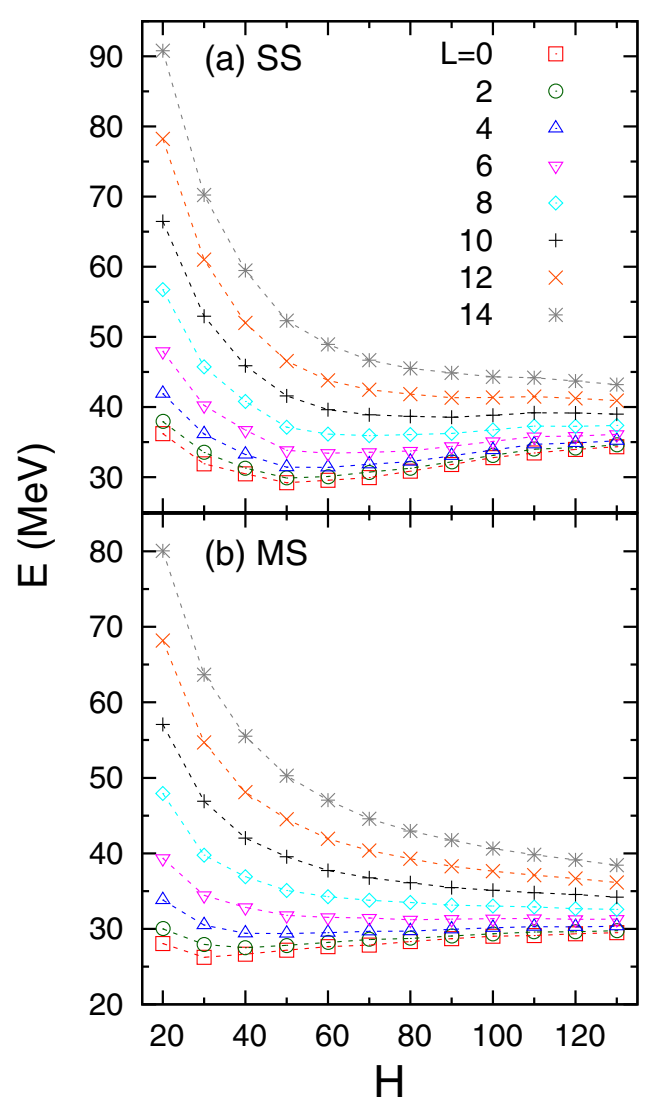

FIG. 3. Energies of LC configurations with the angular momentum $L$ and positive parity. Panel (a) shows the lowest energy calculated in the SS model, whereas (b) shows the lowest energy in the MS model. Although it slightly depends on $L, r_{\text {rms }}$ increases from about 2.28 for $H=20$ to $5.62 \mathrm{fm}$ for $H=130$.

symmetry is imposed, the relative distances calculated with this CG are $D_{12}=2.68, D_{13}=5.70, D_{14}=9.22, D_{23}=3.44$, $D_{24}=6.90, D_{34}=3.81 \mathrm{fm}$, respectively, which are all in very good agreement with those calculated from the LC state (21)

$$
\begin{aligned}
& \left\langle\Phi_{S L M}^{v}(\mathrm{LC})\left|\left(\boldsymbol{r}_{i}-\boldsymbol{r}_{j}\right)^{2}\right| \Phi_{S L M}^{v}(\mathrm{LC})\right\rangle \\
& \quad=\frac{3}{v}\left(1-\delta_{i, j}\right)+\frac{1}{2}\left(S_{i}-S_{j}\right)^{2}\left[1+\frac{L}{H}+\frac{i_{L+1}(H)}{i_{L}(H)}\right] .
\end{aligned}
$$

Next we allow a mixing of various configurations, $\sum_{\kappa} C_{S_{\kappa}^{H}} f_{S_{\kappa}^{H} L M}^{v}(\mathrm{LC}: \mathrm{CG})$, while still keeping $H$ fixed. This calculation, named an MS model, allows us to evaluate the extent to which the energy gain over the SS model is obtained by including the vibrational mode of the LC state. The lowest energy found in the MS model is plotted in panel (b) of Fig. 3. The MS model still presents a local energy minimum at $H \approx 30-40$ for $L=0,2$ and at $H \approx 50$ for $L=4$. Although the minimum is found at $H \approx 80$ for $L=6$, the energy change is very small around that $H$ value. The energy curve of $L=8$ becomes flat with increasing $H$, and no minima appear for higher $L$ values. This suggests that the LC state with large $L$ value is probably not stable against the vibrational degree of freedom even under the LC restriction, but the minimum 


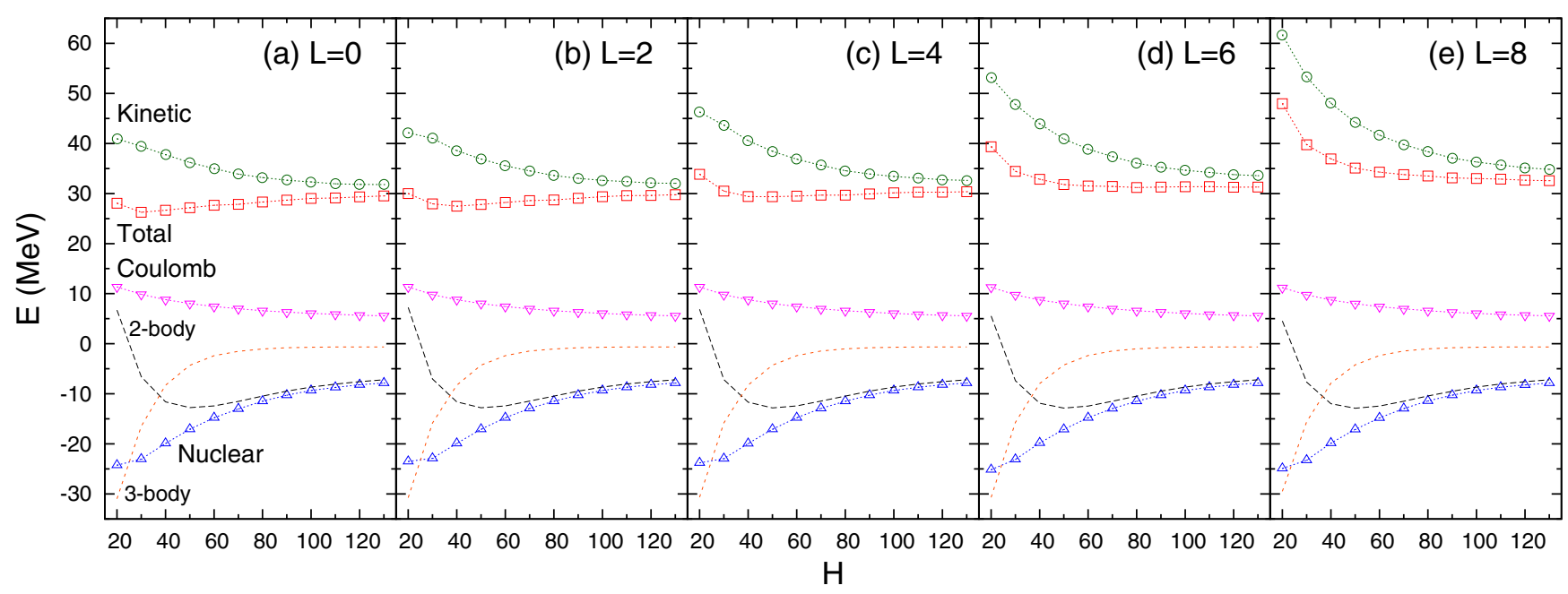

FIG. 4. $H$-dependence of the contributions of the kinetic energy, the nuclear potential, and the Coulomb potential to the lowest energy LC states obtained in the MS model. The nuclear contribution consists of two-body $\left(V_{2 \mathrm{~B}}\right)$ and three-body $\left(V_{3 \mathrm{~B}}\right)$ potential energies. Panels $(\mathrm{a})$, (b), (c), (d), and (e) show $L^{\pi}=0^{+}, 2^{+}, 4^{+}, 6^{+}$, and $8^{+}$cases, respectively.

configuration found in the SS model tends to shift to larger rms size or to break into $\alpha$ particles.

The contributions of the kinetic energy, the nuclear potential $\left(V_{2 \mathrm{~B}}+V_{3 \mathrm{~B}}\right)$, and the Coulomb potential to the lowest energy displayed in Fig. 3(b) are plotted in Fig. 4 for $L^{\pi}=$ $0^{+}, 2^{+}, 4^{+}, 6^{+}, 8^{+}$as a function of $H$. The kinetic energy gives a dominant contribution to the total energy. The nuclear and Coulomb potential contributions change very little with increasing $L$, whereas the kinetic energy contribution considerably depends on $L$. With increasing $L$, the kinetic-energy contribution rapidly increases as $H$ decreases, as expected, and cancels out the nuclear attractive contribution, leaving no energy minimum. It should be noted that the $V_{2 \mathrm{~B}}$ and $V_{3 \mathrm{~B}}$ potentials give an opposite contribution at very small $H$ while the $V_{2 \mathrm{~B}}$ term plays a dominant role in the case of $H>60$.

The CG has the advantage that it can be applied to negative-parity states without any modification. To examine the possibility of finding a negative-parity LC state, we study the $E$ - $H$ diagram for $L^{\pi}=1^{-}$in the same way as the positiveparity case. It turns out that the kinetic energy gets larger and the nuclear potential energy becomes much less attractive, which leads us to the conclusion that no negative-parity $4 \alpha$ LC states exist.

Finally we mix various LC configurations with different $H$ values, $\sum_{H \kappa} C_{S_{\kappa}^{H}} f_{S_{\kappa}^{H} L M}^{v}(\mathrm{LC}: \mathrm{CG})$. This calculation is called an $\mathrm{MH}$ model. The configurations in the range of $H=20,30, \ldots, 130$ are included. The dimension of the Hamiltonian matrix is about 430. The energy gain obtained with the MH model, compared to the SS calculation, is very large, amounting to about $10 \mathrm{MeV}$. Table II lists the result of the lowest LC states obtained in the MH model. The excitation energies, $E_{x}=E+E_{\mathrm{th}}$, of the $0^{+}, 2^{+}, 4^{+}$, and $6^{+}$LC states are predicted to be $32.9,33.7,35.3$, and $37.2 \mathrm{MeV}$, respectively. They follow the $\left(\hbar^{2} / 2 \mathcal{I}\right) L(L+1)$ rule with $\hbar^{2} / 2 \mathcal{I} \approx 0.10$ or $0.12 \mathrm{MeV}$ if the $6^{+}$state is excluded. This parameter is close to that of Ref. [25], but considerably larger than those (0.06-0.08 MeV) estimated in Refs. [17,24,26]. The energies of the second lowest LC states are 20.97, 21.72, 23.16, and $25.37 \mathrm{MeV}$ for $L^{\pi}=0^{+}, 2^{+}, 4^{+}$, and $6^{+}$, respectively.

The contributions of the kinetic energy and the potential energies to $E$ show an interesting contrast. Both contributions of the kinetic energy and the two-body nuclear potential are only weakly dependent on $L$. On the other hand, the contributions of the three-body nuclear and Coulomb potentials alter significantly as a function of $L$; that is, they follow the change of the rms radius that increases with $L$. The $L(L+1)$ rotational spectrum of the $L C$ states is therefore mainly due to the three-body nuclear and Coulomb potentials, which are both long-range pieces of the Hamiltonian. This is in sharp contrast to the rotation of a rigid-body where the kinetic energy should play a primary role in forming the $L(L+1)$ pattern.

Table II lists the $\delta_{2}$ value (42) as well. The limiting value of $\delta_{2}$ with the CG is $-2 L /(2 L+3)$, as shown in Appendix E. The MH model gives $\delta_{2}$ close to that limit for each $L^{+}$state, indicating a large quadrupole deformation. Assuming $\left\langle x^{2}\right\rangle=$ $\left\langle y^{2}\right\rangle$ valid for an axial symmetric shape, the calculated $\delta_{2}$ value suggests the ratio of the major radius to the minor radius of

TABLE II. Energies, given in units of $\mathrm{MeV}$, of the lowest LC states from $4 \alpha$ threshold predicted in the MH model and the contributions from the kinetic (K.E.), two-body potential $\left(V_{2 \mathrm{~B}}\right)$, three-body potential $\left(V_{3 \mathrm{~B}}\right)$, and Coulomb potential $\left(V_{\mathrm{C}}\right)$ energies. The rms radius of ${ }^{16} \mathrm{O}$ is estimated as $\sqrt{r_{\text {rms }}^{2}+1.455^{2}}$ by taking into account the finite size of the $\alpha$ particle.

\begin{tabular}{cccccccc}
\hline \hline$L^{\pi}$ & $E$ & K.E. & \multicolumn{1}{c}{$V_{2 \mathrm{~B}}$} & \multicolumn{1}{c}{$V_{3 \mathrm{~B}}$} & $V_{\mathrm{C}}$ & $r_{\text {rms }}(\mathrm{fm})$ & $\delta_{2}$ \\
\hline $0^{+}$ & 18.46 & 28.17 & -9.62 & -8.54 & 8.46 & 3.47 & 0 \\
$2^{+}$ & 19.31 & 28.08 & -10.24 & -6.59 & 8.07 & 3.67 & -0.554 \\
$4^{+}$ & 20.82 & 28.05 & -10.69 & -4.00 & 7.45 & 4.03 & -0.709 \\
$6^{+}$ & 22.72 & 28.21 & -10.27 & -2.03 & 6.81 & 4.43 & -0.783 \\
\hline \hline
\end{tabular}




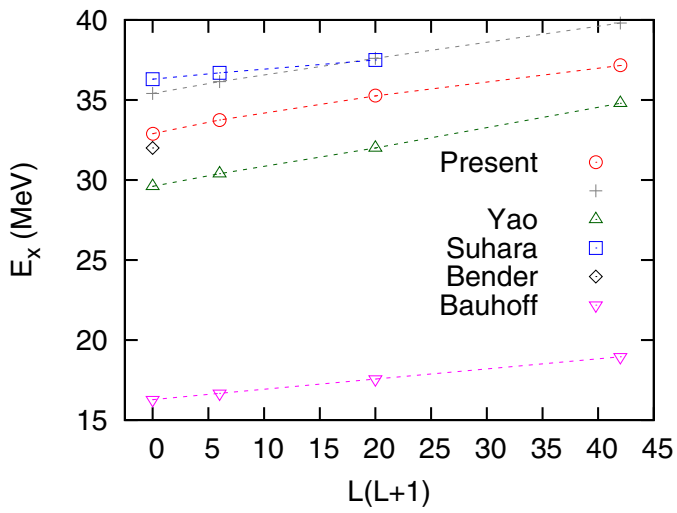

FIG. 5. Comparison of the excitation energies of the $4 \alpha$ LC states as a function of $L(L+1)$. Open circles and crosses denote the lowest and the second lowest LC states obtained in the MH model. Data are taken from Yao [25], Suhara [26], Bender [49], and Bauhoff [48].

the LC state as

$$
\frac{\sqrt{\left\langle y^{2}\right\rangle}}{\sqrt{\left\langle z^{2}\right\rangle}}=\sqrt{\frac{2-\delta_{2}}{2\left(1+\delta_{2}\right)}} \approx 1.69,2.16,2.53
$$

for $L=2,4,6$, respectively. Note that the ratio approaches $\sqrt{L+1}$ in the limit of $\delta_{2} \rightarrow-2 L /(2 L+3)$. If we assume the obtained LC state to have such intrinsic density that is constant inside an axially symmetric spheroid with the quadrupole deformation parameter $\beta$, we may estimate $\beta$ from the following equation:

$$
\frac{\sqrt{\left\langle y^{2}\right\rangle}}{\sqrt{\left\langle z^{2}\right\rangle}}=\frac{1+\sqrt{\frac{5}{16 \pi}} 2 \beta}{1-\sqrt{\frac{5}{16 \pi}} \beta} .
$$

The resulting values of $\beta$ are respectively $0.59,0.88,1.07$ for $L=2,4,6$, indicating very large deformation.

The CG is based on a spherical representation and includes no explicit deformation parameters. Nevertheless the resulting wave functions are found to represent very large deformation. This is primarily made possible by the use of very large $K$ values. A usual approach is to explicitly include some parameters relevant to the deformation. For instance, it is shown in Ref. [26] that the LC state has very large overlap with the rotating state projected from the intrinsically deformed configuration

$$
e^{-\frac{v}{2} R_{x}^{2}-\frac{v^{\prime}}{2} R_{y}^{2}-\frac{v}{2} R_{z}^{2}}
$$

where $R_{x}^{2}$ and $R_{y}^{2}$ are defined in exactly the same way as $R_{z}^{2}$, and $v^{\prime}$ is taken to be much smaller than $v$, typically $0.027 \mathrm{fm}^{-2}$, to embody the shape elongated along the $y$ direction. Note that Eq. (55) is obtained by deforming the hyperradial Gaussian, $e^{-\frac{v}{2} R^{2}}=e^{-\frac{v}{2} \widetilde{\rho} \Lambda \rho}$.

The excitation energies of the LC states obtained by several models are compared in Fig. 5. Except for Brink's $\alpha$-cluster model result [48], the bandhead of the LC states is predicted to be much higher than that speculated in Ref. [17] and has been targeted experimentally [19,21]. Our bandhead energy (the open circle) is close to that estimated by a generator coordinate approach using the Skyrme-Hartree-Fock (HF)+BCS model [49] and falls between those energies calculated by a covariant density functional calculation [25] and a generator coordinate treatment of the Brink wave functions [26]. Our result is very satisfactory in view of the phenomenological treatment of the Hamiltonian. In contrast to a cranked HF calculation [24] that reports the stabilized LC states with $L=13-18$, our calculation finds no local energy minima at the LC configurations rotating with such high angular momenta, consistent with Refs. [25,26]. The second lowest LC states of our calculation are drawn by crosses in the figure. They are in fair agreement with the result of Ref. [26].

\section{CONCLUSIONS}

The single-particle wave function that is angularmomentum projected from the Gaussian wave packet provides spatially localized configurations. We have shown that the linear-chain configuration projected from a product of the Gaussian wave packets can be approximated to high accuracy by the correlated Gaussians if their parameters $A$, $u$, and $K$ are chosen under the condition developed here. Although the correlated Gaussians have been known for the last twenty years, a particular choice of $K$ has led to those configurations which have strongly deformed intrinsic shape but nevertheless are eigenfunctions of the angular momentum.

The present formulation makes it possible to perform calculations of variation-after-projection type. Combined with the advantage that the needed matrix elements can be analytically obtained with the correlated Gaussians, we have studied a system of four $\alpha$ particles in order to examine possible existence of the linear-chain states in ${ }^{16} \mathrm{O}$. The two- $\alpha$ and three- $\alpha$ phenomenological potentials are set to reproduce the ground state and the first excited $2^{+}$state of ${ }^{12} \mathrm{C}$ reasonably well. The energies of the chain configurations are calculated as a function of the angular momentum as well as the size of the four- $\alpha$ system. The full calculation taking into account the vibration of the $\alpha$ particles and the extension of the chain length finds the possibility of chain states with $0^{+}, 2^{+}$, and $4^{+}$. The case with $6^{+}$may be marginal, and no possibility of chain states is observed for $J \geqslant 8$. The bandhead energy of the chain states is about $33 \mathrm{MeV}$ from the ground state of ${ }^{16} \mathrm{O}$. Though those chain states are found to follow a rotational sequence of $J(J+1)$, its physical aspect is significantly different from the case of an ideal rigid-body rotation. The combined effect of the long-range pieces of the Hamiltonian is mainly responsible for its pattern.

One of the important issues is the stability of the linear chain state. To discuss the stability, one has to take account of the coupling of the linear chain configurations with other more general configurations. We have to prepare various configurations and to perform calculations that include them together with the linear chain states. Those configurations can be expressed by the correlated Gaussians that are specified by flexible parameters, $A, u, K$. A work along this direction is interesting and will be reported elsewhere. 


\section{ACKNOWLEDGMENTS}

One of the authors (Y.S.) would like to thank N. Itagaki for useful discussions that were made possible by a visiting program (January 2017) of the Yukawa Institute for Theoretical Physics, Kyoto University (Japan). He also heartily thanks L. Tomio for the generous invitation (September to December 2015) to Universidade Federal do ABC (Brazil), where an early stage of the work was done. This work was in part supported by JSPS KAKENHI Grant No. JP15K05072.

\section{APPENDIX A: GLOSSARY OF ACRONYMS}

\begin{tabular}{lll}
\hline \hline Acronym & \multicolumn{1}{c}{ Full word } & First occurrence \\
\hline CG & correlated Gaussian & Eq. (3) \\
c.m. & center of mass & Sec. III A \\
GWP & Gaussian wave packet & Eq. (1) \\
HO & harmonic-oscillator & Sec. II A \\
LC & linear-chain & Sec. I \\
LPG & locally peaked Gaussian & Eq. (2) \\
MH & mixed $H$ & Sec. IV D \\
MS & mixed $S$ & Sec. IV D \\
rms & root-mean-square & Sec. IV C \\
SG & shifted-Gaussian & Eq. (6) \\
sp & single-particle & Sec. I \\
SS & single $S$ & Sec. IV D \\
VAP & variation after projection & Sec. III C \\
VBP & variation before projection & Sec. III C \\
\hline \hline
\end{tabular}

\section{APPENDIX B: GLOSSARY OF SYMBOLS}

\begin{tabular}{ll}
\hline \hline Symbol & \multicolumn{1}{c}{ Definition } \\
\hline$G_{K}$ & $\left(\frac{\Gamma\left(\kappa+\frac{3}{2}\right)}{2 \sqrt{\pi}}\right)^{\frac{1}{2}}$ \\
$\gamma_{K K^{\prime} L}(x)$ & $\sum_{n=0}^{\min \left(K, K^{\prime}\right)} \frac{K ! K^{\prime} ! \Gamma\left(L+\frac{3}{2}\right)}{n !(K-n) !\left(K^{\prime}-n\right) ! \Gamma\left(n+L+\frac{3}{2}\right)} x^{n}$ \\
$\mathcal{N}_{K L}^{u A}$ & $\frac{1}{(2 K+2 L+1) ! !} \sqrt{\frac{4 \pi(2 L+1) ! !}{\gamma_{K K L}(1)}\left(\frac{\operatorname{det} A}{\pi^{N-1}}\right)^{\frac{3}{4}}}$ \\
& $\times\left(\frac{1}{2} \widetilde{u} A^{-1} u\right)^{-\frac{2 K+L}{2}}$ \\
$G_{K L}(y, z)$ & $\frac{\pi^{\frac{1}{4}}}{\sqrt{2 \gamma_{K K L}(1)}} \sum_{n=0}^{K} \frac{K ! \sqrt{\Gamma\left(L+\frac{3}{2}\right)} y^{K-n}\left(\frac{z}{2}\right)^{2 n+L}}{n !(K-n) ! \Gamma\left(n+L+\frac{3}{2}\right)}$ \\
$b_{l}(x)$ & $\left(i_{l}(x) e^{-x}\right)^{\frac{1}{2}}$ \\
$O_{l}(v s, a k)$ & $\frac{1}{2}\left(\frac{\eta}{2}\right)^{\frac{l}{2}}\left(\frac{2 a}{v+a}\right)^{k}\left(\frac{2 \sqrt{v a}}{v+a}\right)^{l+\frac{3}{2}} \frac{\Gamma\left(k+l+\frac{3}{2}\right)}{b_{l}(\eta) \Gamma\left(l+\frac{3}{2}\right) G_{2 k+l}}$ \\
& $\operatorname{where~} \eta=\frac{1}{2} \nu s^{2}$ \\
$B_{K L}$ & $\frac{4 \pi(2 K+L) !}{(2 K) !(2 K+2 L+1) ! !}$ \\
$g(\alpha, \boldsymbol{e} ; u, A, \boldsymbol{\rho})$ & $\exp \left(-\frac{1}{2} \widetilde{\boldsymbol{\rho}} A \boldsymbol{\rho}+\alpha \boldsymbol{e} \cdot(\tilde{u} \boldsymbol{\rho})\right)$ \\
\hline \hline
\end{tabular}

Here $\Gamma$ is the gamma function.

\section{APPENDIX C: FUNCTION $z i_{l+1}(z) / i_{l}(z)$}

As seen in Eqs. (11) and (28), the function $f_{z}(l)=$ $z i_{l+1}(z) / i_{l}(z)$ appears in the matrix elements involving the

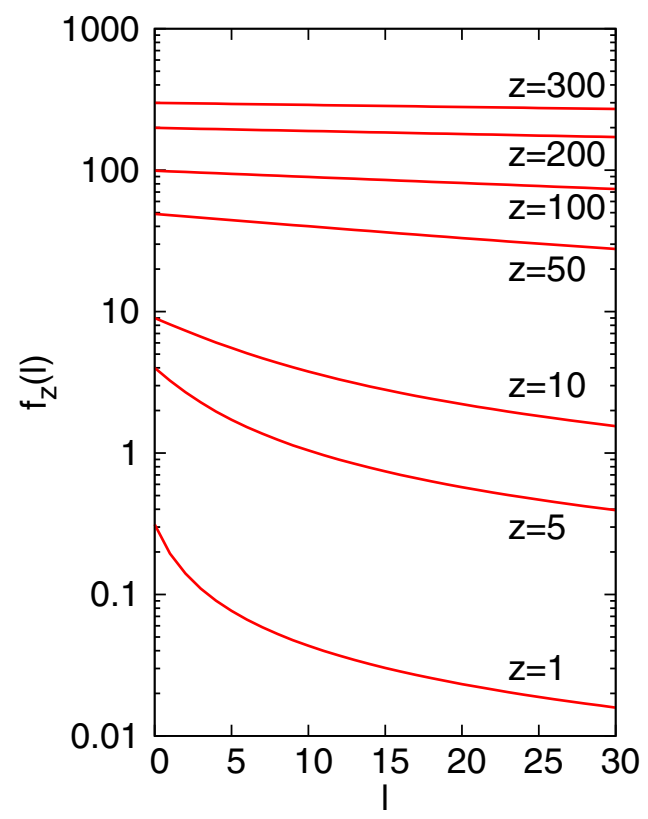

FIG. 6. Plots of $f_{z}(l)$ as a function of $l$ for some values of $z$.

angular-momentum projected GWP. Figure 6 plots $f_{z}(l)$ for some values of $z$. We find numerically that the following inequality holds:

$$
f_{z}(l) \geqslant \sqrt{\left(l+\frac{3}{2}\right)^{2}+z^{2}}-l-\frac{3}{2} .
$$

Actually the right-hand side of the inequality is a good approximation to $f_{z}(l)$. The relative error becomes largest at $l=0$ for any $z$, and its maximum relative error becomes largest, about $8 \%$, at $z \approx 3$. Note that $f_{z}(l) \approx z^{2} /(2 l+3)$ for $z \ll l$, while $f_{z}(l) \approx z-l-\frac{3}{2}$ for $z \gg l$.

If a rigid-body approximation works well, we expect that the rms radius is constant and the kinetic energy is proportional to $l(l+1)$. This is translated to the following behavior of $f_{z}(l): f_{z}(l)+l+z$ is $l$ independent, whereas $f_{z}(l)+l-z$ is proportional to $l(l+1)$. A numerical check indicates that this expectation very much depends on $z$. For $z=20$, the rigid-body approximation is reasonable up to $l=20$. With increasing $z$ the approximation works better up to larger $l$. For example, with $z=40$, the approximation works well up to about $l=40$, and with $z=60$ it significantly improves.

\section{APPENDIX D: MATRIX ELEMENT OF $\tilde{\rho} \Omega \rho$}

The operator $\tilde{\rho} \Omega \rho$ is scalar and quadratic in $\rho$, where the matrix $\Omega$ may not be necessarily symmetric. We obtain its matrix element between the CGs following the procedure of Ref. [8]:

$$
\begin{aligned}
& \left\langle f_{K L M}^{u A}|\tilde{\boldsymbol{\rho}} \Omega \boldsymbol{\rho}| f_{K^{\prime} L M}^{v B}\right\rangle \\
& =\frac{\mathcal{N}_{K L}^{u A} \mathcal{N}_{K^{\prime} L}^{v B}}{B_{K L} B_{K^{\prime} L}} \iint d \boldsymbol{e} d \boldsymbol{e}^{\prime} Y_{L M}^{*}(\hat{\boldsymbol{e}}) Y_{L M}\left(\hat{\boldsymbol{e}^{\prime}}\right)\left(\frac{d^{2 K+L+2 K^{\prime}+L}}{d \alpha^{2 K+L} d \alpha^{\prime 2 K^{\prime}+L}}\right. \\
& \left.\quad \times\left\langle g(\alpha, \boldsymbol{e} ; u, A, \boldsymbol{\rho})|\tilde{\boldsymbol{\rho}} \Omega \rho| g\left(\alpha^{\prime}, \boldsymbol{e}^{\prime} ; v, B, \boldsymbol{\rho}\right)\right\rangle\right)_{\alpha=\alpha^{\prime}=0}^{.} \text {(D1) }
\end{aligned}
$$


The matrix element between the generating functions reads

$$
\begin{aligned}
& \left\langle g(\alpha, \boldsymbol{e} ; u, A, \boldsymbol{\rho})|\tilde{\rho} \Omega \rho| g\left(\alpha^{\prime}, \boldsymbol{e}^{\prime} ; v, B, \boldsymbol{\rho}\right)\right\rangle \\
& =\left(\frac{\pi^{N-1}}{\operatorname{det} C}\right)^{\frac{3}{2}} e^{p \alpha^{2}+p^{\prime} \alpha^{\prime 2}+q \alpha \alpha^{\prime} \boldsymbol{e} \cdot \boldsymbol{e}^{\prime}} \\
& \quad \times\left[3 R(\Omega)+P(\Omega) \alpha^{2}+P^{\prime}(\Omega) \alpha^{\prime 2}+Q(\Omega) \alpha \alpha^{\prime} \boldsymbol{e} \cdot \boldsymbol{e}^{\prime}\right],
\end{aligned}
$$

where $C=\frac{1}{2}(A+B)$ and the various coefficients are

$$
\begin{aligned}
p & =\frac{1}{4} \tilde{u} C^{-1} u, \quad p^{\prime}=\frac{1}{4} \widetilde{v} C^{-1} v, \\
q & =\frac{1}{4}\left(\tilde{u} C^{-1} v+\widetilde{v} C^{-1} u\right), \\
P(\Omega) & =\frac{1}{4} \tilde{u} C^{-1} \Omega C^{-1} u, \quad P^{\prime}(\Omega)=\frac{1}{4} \widetilde{v} C^{-1} \Omega C^{-1} v, \\
Q(\Omega) & =\frac{1}{4}\left(\widetilde{u} C^{-1} \Omega C^{-1} v+\widetilde{v} C^{-1} \Omega C^{-1} u\right), \\
R(\Omega) & =\frac{1}{2} \operatorname{Tr} C^{-1} \Omega .
\end{aligned}
$$

Performing the differentiation and integration in Eq. (D1) leads to the following result:

$$
\begin{aligned}
\left\langle f_{K L M}^{u A}|\tilde{\rho} \Omega \rho| f_{K^{\prime} L M}^{v B}\right\rangle & \frac{1}{\sqrt{\gamma_{K K L}(1) \gamma_{K^{\prime} K^{\prime} L}(1)}} \bar{t}^{\frac{L}{2}} \\
& \times\left(\frac{\sqrt{\operatorname{det} A B}}{\operatorname{det} C}\right)^{\frac{3}{2}}\left(\frac{\tilde{u} C^{-1} u}{\widetilde{u} A^{-1} u}\right)^{K}\left(\frac{\widetilde{v} C^{-1} v}{\widetilde{v} B^{-1} v}\right)^{K^{\prime}} \\
& \times \sum_{n=0}^{\min \left(K, K^{\prime}\right)} \frac{K ! K^{\prime} ! \Gamma\left(L+\frac{3}{2}\right)}{n !(K-n) !\left(K^{\prime}-n\right) ! \Gamma\left(n+L+\frac{3}{2}\right)} t^{n} \\
& \times\left[3 R(\Omega)+(K-n) \frac{P(\Omega)}{p}+\left(K^{\prime}-n\right) \frac{P^{\prime}(\Omega)}{p^{\prime}}\right. \\
& \left.+(L+2 n) \frac{Q(\Omega)}{q}\right],
\end{aligned}
$$

where

$$
t=\frac{\left(\tilde{u} C^{-1} v\right)^{2}}{\left(\widetilde{u} C^{-1} u\right)\left(\widetilde{v} C^{-1} v\right)}, \quad \bar{t}=\frac{\left(\widetilde{u} C^{-1} v\right)^{2}}{\left(\widetilde{u} A^{-1} u\right)\left(\widetilde{v} B^{-1} v\right)} .
$$

The diagonal matrix element takes a simple form:

$$
\begin{aligned}
& \left\langle f_{K L M}^{u A}|\tilde{\rho} \Omega \rho| f_{K L M}^{u A}\right\rangle \\
& \quad=\frac{3}{2} \operatorname{Tr} A^{-1} \Omega+(2 K+L) \frac{\tilde{u} A^{-1} \Omega A^{-1} u}{\tilde{u} A^{-1} u} .
\end{aligned}
$$

The matrix element of $r_{i j}^{2}$ is obtained by putting $\Omega=\omega^{(i j)} \widetilde{\left.\omega^{(i j)}\right)}$. The formula (32) for $R^{2}$ is obtained by replacing $\Omega$ with $\Lambda$ in the above equation.

In some cases one may want to calculate the mean deviation of $r_{i j}^{2}$ from its mean value, i.e., $\left\langle\left(r_{i j}^{2}-D_{i j}^{2}\right)^{2}\right\rangle=\left\langle r_{i j}^{4}\right\rangle-D_{i j}^{4}$. It is calculated by using the approximation

$$
\left\langle f_{K L M}^{u A}\left|e^{-\epsilon \tilde{\boldsymbol{\rho}} \Omega \rho}\right| f_{K L M}^{u A}\right\rangle \approx 1-\epsilon D_{i j}^{2}+\frac{\epsilon^{2}}{2}\left\langle r_{i j}^{4}\right\rangle+\cdots
$$

The left-hand side of this equation is nothing but the expectation value of the Gaussian-type potential. By taking a small $\epsilon$ value, $\left\langle r_{i j}^{4}\right\rangle$ is easily obtained.

\section{APPENDIX E: MATRIX ELEMENT OF QUADRUPOLE MOMENTS}

We consider the matrix element

$$
\left\langle f_{K L M}^{u A}\left|R_{z}^{2}\right| f_{K^{\prime} L M}^{v B}\right\rangle=\left\langle f_{K L M}^{u A}|\tilde{\zeta} \Lambda \zeta| f_{K^{\prime} L M}^{v B}\right\rangle,
$$

where $\zeta=\left(\zeta_{i}\right)$ is the $(N-1)$-dimensional column vector comprising the $z$ component of $\boldsymbol{\rho}_{i}$. Although we need the case with $M=L$, we here proceed for a general case. The basic step for calculating the matrix element is the same as that of $\tilde{\rho} \Lambda \rho$. The matrix element between the generating functions is easily obtained because they are factorized in $x, y, z$ components:

$$
\begin{aligned}
& \left\langle g(\alpha, \boldsymbol{e} ; u, A, \boldsymbol{\rho})|\tilde{\zeta} \Lambda \zeta| g\left(\alpha^{\prime}, \boldsymbol{e}^{\prime} ; v, B, \boldsymbol{\rho}\right\rangle\right. \\
& =\left(\frac{\pi^{N-1}}{\operatorname{det} C}\right)^{\frac{3}{2}} e^{p \alpha^{2}+p^{\prime} \alpha^{\prime 2}+q \alpha \alpha^{\prime} \boldsymbol{e} \cdot \boldsymbol{e}^{\prime}} \\
& \quad \times\left[R(\Lambda)+P(\Lambda) \alpha^{2} e_{z}^{2}+P^{\prime}(\Lambda) \alpha^{\prime 2} e_{z}^{\prime 2}+Q(\Lambda) \alpha \alpha^{\prime} e_{z} e_{z}^{\prime}\right]
\end{aligned}
$$

where $e_{z}$ is the $z$ component of the unit vector $\boldsymbol{e}, e_{z}=$ $\sqrt{4 \pi / 3} Y_{10}(\hat{\boldsymbol{e}})$, and likewise $e_{z}^{\prime}=\sqrt{4 \pi / 3} Y_{10}\left(\hat{\boldsymbol{e}}^{\prime}\right)$. The matrix element (E2) takes a form similar to that of Eq. (D2), but because of the difference in the tensor character of $\widetilde{\zeta} \Lambda \zeta$, it contains explicit dependence on $e_{z}$ and $e_{z}^{\prime}$ as well. The manipulation needed to obtain the desired matrix element thus involves a slightly lengthy procedure compared to the case of $\tilde{\rho} \Lambda \rho$, leading to the following result:

$$
\begin{aligned}
\left\langle f_{K L M}^{u A}|\tilde{\zeta} \Lambda \zeta| f_{K^{\prime} L M}^{v B}\right\rangle & \frac{1}{\sqrt{\gamma_{K K L}(1) \gamma_{K^{\prime} K^{\prime} L}(1)}} \bar{t}^{\frac{L}{2}} \\
& \times\left(\frac{\sqrt{\operatorname{det} A B}}{\operatorname{det} C}\right)^{\frac{3}{2}}\left(\frac{\tilde{u} C^{-1} u}{\widetilde{u} A^{-1} u}\right)^{K}\left(\frac{\widetilde{v} C^{-1} v}{\widetilde{v} B^{-1} v}\right)^{K^{\prime}} \\
& \times \sum_{n=0}^{\min \left(K, K^{\prime}\right)} \frac{K ! K^{\prime} ! \Gamma\left(L+\frac{3}{2}\right)}{n !(K-n) !\left(K^{\prime}-n\right) ! \Gamma\left(n+L+\frac{3}{2}\right)} t^{n} \\
& \times\left[R(\Lambda)+(K-n) \frac{P(\Lambda)}{p} c_{L M}^{(0)}+\left(K^{\prime}-n\right) \frac{P^{\prime}(\Lambda)}{p^{\prime}} c_{L M}^{(0)}\right. \\
& \left.+\frac{Q(\Lambda)}{q} c_{n L M}^{(1)}\right],
\end{aligned}
$$

where

$$
\begin{aligned}
c_{L M}^{(0)} & =\frac{1}{2 L+3}+\frac{2\left(L^{2}-M^{2}\right)}{(2 L-1)(2 L+3)}, \\
c_{n L M}^{(1)} & =2 n c_{L M}^{(0)}+\frac{L^{2}-M^{2}}{2 L-1} .
\end{aligned}
$$

The diagonal matrix element with $M=L$ reads

$$
\begin{aligned}
& \left\langle f_{K L L}^{u A}|\widetilde{\zeta} \Lambda \zeta| f_{K L L}^{u A}\right\rangle \\
& \quad=\frac{1}{2} \operatorname{Tr} A^{-1} \Lambda+\frac{2 K}{2 L+3} \frac{\tilde{u} A^{-1} \Lambda A^{-1} u}{\widetilde{u} A^{-1} u}
\end{aligned}
$$

and therefore $\delta_{2}$ defined by Eq. (42) is found to be

$$
\delta_{2}=-\frac{2 L}{2 L+3} \frac{2 K+L+\frac{3}{2}}{2 K+L+\frac{3}{2} \kappa}
$$


with

$$
\kappa=\frac{\widetilde{u} A^{-1} u}{\widetilde{u} A^{-1} \Lambda A^{-1} u} \operatorname{Tr} A^{-1} \Lambda
$$

For the choice of $A=a v \Lambda$, Eq. (34), used to approximate the LC configuration with the CG, $\kappa$ reduces to $N-1$ independent of $u$. Then $\delta_{2}$ approaches $-2 L /(2 L+3)$ in the limit of $K \rightarrow \infty$.
[1] D. M. Brink, in Proceedings of the International School of Physics, "Enrico Fermi”, Course XXXVI, 1965, edited by C. Bloch (Academic, New York, 1966), p. 247.

[2] T. Neff and H. Feldmeier, Eur. Phys. J. Spec. Top. 156, 69 (2008).

[3] Y. Kanada-En'yo, M. Kimura, and A. Ono, Prog. Theor. Exp. Phys. 2012, 01A202 (2012).

[4] K. Varga and Y. Suzuki, Phys. Rev. C 52, 2885 (1995).

[5] Y. Suzuki and K. Varga, Stochastic Variational Approach to Quantum-Mechanical Few-Body Problems, Lecture Notes in Physics (Springer, Berlin, 1998), Vol. 54.

[6] S. F. Boys, Proc. R. Soc. London A 258, 402 (1960).

[7] K. Singer, Proc. R. Soc. London A 258, 412 (1960).

[8] Y. Suzuki, J. Usukura, and K. Varga, J. Phys. B 31, 31 (1998).

[9] K. Varga, J. Usukura, and Y. Suzuki, Phys. Rev. Lett. 80, 1876 (1998).

[10] Y. Suzuki and J. Usukura, Nucl. Instrum. Methods B 171, 67 (2000).

[11] H. Nemura, Y. Akaishi, and Y. Suzuki, Phys. Rev. Lett. 89, 142504 (2002).

[12] W. Horiuchi, Y. Suzuki, and K. Arai, Phys. Rev. C 85, 054002 (2012).

[13] W. Horiuchi and Y. Suzuki, Phys. Rev. C 89, 011304(R) (2014).

[14] D. Mikami, W. Horiuchi, and Y. Suzuki, Phys. Rev. C 89, 064303 (2014).

[15] J. Mitroy, S. Bubin, W. Horiuchi, Y. Suzuki, L. Adamowicz, W. Cencek, K. Szalewicz, J. Komasa, D. Blume, and K. Varga, Rev. Mod. Phys. 85, 693 (2013).

[16] H. Morinaga, Phys. Rev. 101, 254 (1956).

[17] P. Chevallier, F. Schebling, G. Goldring, I. Plesser, and M. W. Sachs, Phys. Rev. 160, 827 (1967).

[18] Y. Suzuki, H. Horiuchi, and K. Ikeda, Prog. Theor. Phys. 47, 1517 (1972).

[19] M. Freer, N. M. Clarke, N. Curtis, B. R. Fulton, S. J. Hall, M. J. Leddy, J. S. Pople, G. Tungate, R. P. Ward, P. M. Simmons, W. D. M. Rae, S. P. G. Chappell, S. P. Fox, C. D. Jones, D. L. Watson, G. J. Gyapong, S. M. Singer, W. N. Catford, and P. H. Regan, Phys. Rev. C 51, 1682 (1995).

[20] M. Freer, M. P. Nicoli, S. M. Singer, C. A. Bremner, S. P. G. Chappell, W. D. M. Rae, I. Boztosun, B. R. Fulton, D. L. Watson, B. J. Greenhalgh, G. K. Dillon, R. L. Cowin, and D. C. Weisser, Phys. Rev. C 70, 064311 (2004).

[21] N. Curtis, S. Almaraz-Calderon, A. Aprahamian, N. I. Ashwood, M. Barr, B. Bucher, P. Copp, M. Couder, X. Fang, M. Freer, G. Goldring, F. Jung, S. R. Lesher, W. Lu, J. D. Malcolm, A. Roberts, W. P. Tan, C. Wheldon, and V. A. Ziman, Phys. Rev. C 94, 034313 (2016).

[22] T. Suhara and Y. Kanada-En'yo, Phys. Rev. C 82, 044301 (2010).

[23] T. Suhara and Y. Kanada-En'yo, Phys. Rev. C 84, 024328 (2011).

[24] T. Ichikawa, J. A. Maruhn, N. Itagaki, and S. Ohkubo, Phys. Rev. Lett. 107, 112501 (2011).

[25] J. M. Yao, N. Itagaki, and J. Meng, Phys. Rev. C 90, 054307 (2014).
[26] T. Suhara, Y. Funaki, B. Zhou, H. Horiuchi, and A. Tohsaki, Phys. Rev. Lett. 112, 062501 (2014).

[27] P. W. Zhao, N. Itagaki, and J. Meng, Phys. Rev. Lett. 115, 022501 (2015).

[28] Y. Iwata, T. Ichikawa, N. Itagaki, J. A. Maruhn, and T. Otsuka, Phys. Rev. C 92, 011303(R) (2015).

[29] G. Royer, G. Ramasamy, and P. Eudes, Phys. Rev. C 92, 054308 (2015)

[30] M. Freer, J. D. Malcolm, N. L. Achouri, N. I. Ashwood, D. W. Bardayan, S. M. Brown, W. N. Catford, K. A. Chipps, J. Cizewski, N. Curtis, K. L. Jones, T. Munoz-Britton, S. D. Pain, N. Soić, C. Wheldon, G. L. Wilson, and V. A. Ziman, Phys. Rev. C 90, 054324 (2014).

[31] A. Fritsch, S. Beceiro-Novo, D. Suzuki, W. Mittig, J. J. Kolata, T. Ahn, D. Bazin, F. D. Becchetti, B. Bucher, Z. Chajecki, X. Fang, M. Febbraro, A. M. Howard, Y. Kanada-En'yo, W. G. Lynch, A. J. Mitchell, M. Ojaruega, A. M. Rogers, A. Shore, T. Suhara, X. D. Tang, R. Torres-Isea, and H. Wang, Phys. Rev. C 93, 014321 (2016).

[32] H. Yamaguchi et al., Phys. Lett. B 766, 11 (2017).

[33] M. Abramowitz and I. A. Stegun, Handbook of Mathmatical Functions with Formulas, Graphs, and Mathematical Tables (Dover, Mineola, NY, 1970).

[34] R. G. Lovas, R. J. Liotta, A. Insolia, K. Varga, and D. S. Delion, Phys. Rep. 294, 265 (1998).

[35] K. Varga, R. G. Lovas, and R. J. Liotta, Phys. Rev. Lett. 69, 37 (1992).

[36] K. Varga and R. G. Lovas, Nucl. Phys. A 550, 421 (1992).

[37] M. Włoch, D. J. Dean, J. R. Gour, M. Hjorth-Jensen, K. Kowalski, T. Papenbrock, and P. Piecuch, Phys. Rev. Lett. 94, 212501 (2005).

[38] P. Maris, J. P. Vary, and A. M. Shirokov, Phys. Rev. C 79, 014308 (2009)

[39] Y. Kanada-En'yo, Phys. Rev. Lett. 81, 5291 (1998).

[40] H. Flocard, P.-H. Heenen, S. J. Krieger, and M. S. Weiss, Prog. Theor. Phys. 72, 1000 (1984).

[41] P. Ring and P. Schuck, The Nuclear Many-Body Problem (Springer, New York, 1980).

[42] S. G. Nilsson and I. Ragnarsson, Shapes and Shells in Nuclear Structure (Cambridge University Press, Cambridge, 1995)

[43] R. B. Wiringa, S. C. Pieper, J. Carlson, and V. R. Pandharipande, Phys. Rev. C 62, 014001 (2000).

[44] H. Suno, Y. Suzuki, and P. Descouvemont, Phys. Rev. C 94, 054607 (2016).

[45] S. Saito, Suppl. Prog. Theor. Phys. 62, 11 (1977).

[46] E. Nielsen, D. V. Fedorov, A. S. Jensen, and E. Garrido, Phys. Rep. 347, 373 (2001).

[47] Y. Suzuki, Prog. Theor. Exp. Phys. 2015, 043 D05 (2015).

[48] W. Bauhoff, H. Schultheis, and R. Schultheis, Phys. Rev. C 29, 1046 (1984).

[49] M. Bender and P.-H. Heenen, Nucl. Phys. A 713, 390 (2003). 